\title{
A PRIORI AND POSTERIORI ERROR ESTIMATES OF LEGENDRE GALERKIN SPECTRAL METHODS FOR GENERAL ELLIPTIC OPTIMAL CONTROL PROBLEMS
}

\author{
Zuliang Lu, Fei Huang, Li Lin, Fei Cai And Yin Yang
}

\begin{abstract}
In this paper, the Legendre Galerkin spectral method is applied to solve the constrained optimal control problems governed by general elliptic equations. Under some reasonable assumptions, by using the orthogonal projection operator, we derive a priori error estimates for the spectral approximation of optimal control problems. Then, we obtain a posteriori error estimates for both the state and the control approximation, where we use the $L^{2}$-norm for estimating the control approximation error, and the $H^{1}$-norm or $L^{2}$-norm for the state and co-state approximation error. Finally, some numerical experiments are presented to test our theoretical results.
\end{abstract}

Mathematics subject classification (2010): 49J20, 65N30.

Keywords and phrases: General optimal control problems, Legendre Galerkin spectral method, a priori error estimate, a posteriori error estimates.

\section{REFERENCES}

[1] R. A. Adams, Sobolev Spaces, Academic Press, New York, San Francisco, London, 1975.

[2] S. Collis And M. Heinkenschloss, Analysis of the streamline upwind/Petrov Galerkin method applied to the solution of optimal control problems, CAAM TR02-01, (2002).

[3] Y. CHEN, Y. HUANG, W. LIU AND N. YAN, Error estimates and superconvergence of mixed finite element methods for convex optimal control problems, J. Sci. Comp., 3 (2010), pp. 382-403.

[4] Y. Chen, F. HuAng, N. YI AND W. LiU, A Legendre-Galerkin spectral method for optimal control problems governed by stokes equations, SIAM J. Numer. Anal., 4 (2011), pp. 1625-1648.

[5] Y. CHEN AND Z. LU, High efficient and accuracy numerical methods for optimal control problems, Science Press, Beijing, 2015.

[6] Y. CHEN AND Z. LU, Error estimates of fully discrete mixed finite element methods for semilinear quadratic parabolic optimal control problems, Comput. Methods Appl. Mech. Engrg., 2010 (2010), pp. 1415-1423.

[7] Y. CHEN, Z. LU AND R. Guo, Error estimates of triangular mixed finite element methods for quasilinear optimal control problems, Frontiers Math. China, 1 (2012), pp. 397-413.

[8] Y. Chen, N. Yi AND W. LiU, A Legendre Galerkin spectral method for optimal control problems governed by elliptic equations, SIAM J. Numer. Anal., 5 (2008), pp. 2254-2275.

[9] S. CHOU AND X. YE, Unified analysis of finite volume methods for second order elliptic problems, SIAM J. Numer. Anal., 45 (2007), pp. 1639-1653.

[10] C. Canuto, M. Y. Hussaini, A. Quarteroni and T. A. Zang, Spectral methods in fluid dynamics, Springer-Verlag, Berlin, 1988.

[11] R. E. EwING, T. LIN AND Y. LIN, On the accuracy of the finite volume element method based on piecewise linear polynomials, SIAM J. Numer. Anal., 6 (2002), pp. 1865-1888.

[12] R. Ghanem AND H. SiSS AOUI, A posteriori error estimate by a spectral method of an elliptic optimal control problem, J. Comput. Math. Optim., 24 (2006), pp. 111-125.

[13] F. HUANG AND Y. CHEN, Error estimates for spectral approximation of elliptic control problems with integral state and control constraints, Comput. Math. Appl., 68 (2014), pp. 789-803. 
[14] O. A. LANyzhenskaya And N. N. URaĹtseva, Linear and quasiliear elliptic equations, Academic Press, New York, 1968.

[15] R. Li, W. LIU, H. MA AND T. TANG, Adaptive finite element approximation for distributed elliptic optimal control problems, SIAM J. Control Optim., 41 (2002), pp. 1321-1349.

[16] J. L. Lions, Optimal control of systems governed by partial differential equations, Springer-Verlag, Berlin, 1971.

[17] W. LIU AND D. TIBA, Error estimates for the finite element approximation of a class of nonlinear optimal control problems, J. Numer. Func. Optim., 22 (2001), pp. 935-972.

[18] X. Luo, Y. Y. Chen, Huang And T. Hou, Some error estimates of finite volume element method for parabolic optimal control problems, Optim. Control Appl. Methods, 2 (2014), pp. 145-165.

[19] J. SHEN, Efficient spectral-Galerkin methed Direct solvers of second and fourth order equations using Legendre polynomials, SIAM J. Sci. Comput., 15 (1994), pp. 1489-1505.

[20] T. Sun, Discontinuous Galerkin finite element method with interior penalties for convection diffusion optimal control problem, Int. J. Numer. Anal. Mod., 7 (2010), pp. 87-107.

[21] N. YAN AND Z. ZHOU, A priori and a posteriori error estimates of streamline diffusion finite element method for optimal control problem governed by convection dominated diffusion equation, Numer. Math. Theory Me. Appl., 1 (2008), pp. 297-320.

[22] J. ZHOU AND D. YANG, Legendre Galerkin spectral methods for optimal control problems with integral constraint for state in one dimension, Comput. Optim. Appl., 61 (2015), pp. 1-24. 